in vitro, l'acquisizione di PA conduce quasi sempre a una colonizzazione cronica per mancata eradicazione del microrganismo. Numerose evidenze in vivo e in vitro suggeriscono che PA cresce nelle vie aeree del paziente con fc sotto forma di biofilm.

I biofilm batterici, che possono essere definiti come comunità di batteri adesi ad una superficie ed immersi in una matrice esopolisaccaridica, presentano una architettura caratteristica e proprietà fenotipiche molto diverse rispetto ai microrganismi in fase planctonica. Una di queste è rappresentata dalla minore sensibilità ai farmaci antimicrobici; pertanto, anche in individui immunocompetenti, le infezioni associate a biofilm sono persistenti e/o ricorrenti.

Queste osservazioni sono in conflitto con la pratica del Laboratorio di microbiologia clinica, in cui i convenzionali test di sensibilità utilizzano cellule batteriche in fase planctonica. Le MIC cosi' misurate, riferimento per il clinico per eventuali scelte terapeutiche, potrebbero non essere predittive dell'efficacia di un antibiotico nei confronti degli stessi batteri in forma sessile in vivo. Appare, pertanto, opportuno e necessario sviluppare e standardizzare metodi per la determinazione dell'attività degli agenti antimicrobici verso il biofilm, affinché i risultati dei test di sensibilità abbiano un impatto sempre migliore sull'outcome clinico.

\title{
S6.4
}

\section{CRITICITÀ NELLA DETERMINAZIONE DELLA SENSIBILITÀ AGLI AGENTI ANTIMICROBICI}

\section{Fiscarelli E.}

\author{
Laboratorio di Microbiologia \\ - Alta Specializzazione in Microbiologia della Fibrosi \\ Cistica, Ospedale Pediatrico Bambino Gesu' IRCCS, \\ Piazza S.Onofrio 4, 00165 Roma
}

Nella pratica clinica, è ormai consolidato l'uso di un test di sensibilità per identificare un appropriato agente antimicrobico per il trattamento di un processo infettivo. Nella maggior parte dei casi, trovare che il ceppo batterico isolato dal focus di infezione è sensibile ad uno specifico antibiotico, è indice dell'efficacia di quel farmaco.Una eccezione è rappresentata dal trattamento delle infezioni polmonari in pazienti con Fibrosi cistica (fc). Pseudomonas aeruginosa (PA) è il patogeno opportunista isolato con maggior frequenza dall'espettorato di soggetti con fc in tutti i gruppi di età. Nonostante trattamenti anche molto aggressivi con combinazioni di antimicrobici con dimostrata potenza 